\title{
What Does Someone's Gender Identity Signal to Employers?*
}

\author{
Hannah Van Bormi, Marlot Dhoop ${ }^{\mathrm{ii}}$, Allien Van Ackerii, and Stijn Baert ${ }^{\mathrm{iv}}$ \\ Structured abstract
}

Purpose - The purpose of this paper is to explore the mechanisms underlying hiring discrimination against transgender men.

Design/methodology/approach - The authors conduct a scenario experiment with final-year business students in which fictitious hiring decisions are made about transgender or cisgender male job candidates. More importantly, these candidates are scored on statements related to theoretical reasons for hiring discrimination given in the literature. The resulting data are analysed using a bivariate analysis. Additionally, a multiple mediation model is run.

Findings - Suggestive evidence is found for co-worker and customer taste-based discrimination, but not for employer taste-based discrimination. In addition, results show that transgender men are perceived as being in worse health, being more autonomous and assertive, and have a lower probability to go on parental leave, compared with cisgender men, revealing evidence for (positive and negative) statistical discrimination.

Social implications - Targeted policy measures are needed given the substantial labour market discrimination against transgender individuals measured in former studies. However, to combat this discrimination effectively, one needs to understand its underlying mechanisms. This study provides the first comprehensive exploration of these mechanisms.

Originality/value - This study innovates in being one of the first to explore the relative empirical importance of dominant (theoretical) explanations for hiring discrimination against transgender men. Thereby, the authors take the logical next step in the literature on labour market discrimination against transgender individuals.

Keywords: transgender men, fictitious hiring decisions, theories of discrimination, signalling, scenario experiment, risk aversion.

JEL: J15, J71, J16, J24, J23.

\footnotetext{
* Acknowledgements. We thank Joz Motmans, Marion Wasserbauer, Simon Amez, Brecht Neyt, and Philippe Sterkens for their comments which helped us to improve this study substantially.

i Corresponding author. Ghent University. Sint-Pietersplein 6, 9000 Ghent, Belgium. Hannah.VanBorm@UGent.be.

ii Gent University.

iii Gent University.

${ }^{\text {iv }}$ Ghent University, Ghent, Belgium. Research Foundation - Flanders. University of Antwerp, Antwerp, Belgium. Université Catholique de Louvain, Louvain-la-Neuve, Belgium. IZA, Bonn, Germany. GLO. IMISCOE, Rotterdam, the Netherlands.
} 


\section{Introduction}

Prior economic research has shown that one's gender identity notably affects people's labour market outcomes (Grant et al., 2011; Motmans et al., 2009; Motmans et al., 2017; OECD, 2019; Rainey et al., 2015). ${ }^{1}$ More precisely, survey research conducted in the United States, the European Union, the United Kingdom, and Belgium showed that a significant number of transgender individuals has experienced unfavourable treatment with respect to hiring chances (Badgett et al., 2007; FRA, 2014; Grant et al., 2011; James et al., 2016; Motmans et al., 2009; Motmans et al., 2017; Valfort, 2017), promotion opportunities (Badgett et al., 2007; Grant et al., 2011; James et al., 2016; Motmans et al., 2009), dismissal decisions (Badgett et al., 2007; Grant et al., 2011; James et al., 2016; Motmans et al., 2009; Schilt and Wiswall, 2008), and wage outcomes (Schilt and Wiswall, 2008; Valfort, 2017). ${ }^{2}$ This self-reported discrimination against transgender individuals seemed to be more severe than similar forms of discrimination towards sexual minorities and other gender minorities (Grant et al., 2011). In addition, research that uses (quasi-)experimental methods (i.e. audit studies and correspondence tests) to measure labour market discrimination towards transgender individuals in a more direct way found similar negative results with respect to employment opportunities (Bardales, 2013; Granberg et al., 2019; Make The Road New York, 2010; Rainey et al., 2015) and wages (Carpenter et al., 2016; Geijtenbeek and Plug, 2015). ${ }^{3}$

In the economic literature, there are two dominant theoretical models that offer an explanation for the occurrence of labour market discrimination towards transgender persons: Becker's (1957) model of taste-based discrimination and Arrow's (1973) model of statistical discrimination. On the one hand, the model of taste-based discrimination (Becker, 1957) implies that discrimination towards transgender individuals is driven by negative attitudes (i.e. transphobia) towards them internalised by employers (employer discrimination), co-workers (co-worker discrimination), or customers (customer discrimination), which, in turn, might lead employers (co-workers) ((customers)) to have a distaste towards interacting with them. ${ }^{4}$ On the other hand, the model of statistical discrimination (Arrow, 1973) implies that discrimination towards transgender individuals is driven by (potentially erroneous) stereotypes regarding their productivity. There is one stereotype, in particular, that may act as an explanation for the unfavourable treatment of transgender persons found in the literature. That is, statistical discrimination might be particularly rooted in the health-related stigma of transgender individuals (Reed et al., 2015; Van Borm and Baert, 2018). In the scientific literature, transgender people are often associated with having an elevated risk for mental issues (and suicide) and HIV (Bockting et al., 2013; Clements-Nolle et al., 2006; Drydakis, 2017a, 2017b), and, as a result, could be perceived as less productive. In turn, this could lead to less favourable

\footnotetext{
1 'Gender identity' is defined as 'a person's deeply felt internal and individual experience of gender, which may or may not correspond with the sex assigned to them at birth' (UNESCO, 2016, p.8).

2 'Transgender' is defined as 'a person whose gender identity differs from their sex assigned at birth' (UNESCO, 2016, p.10)

${ }^{3}$ Similar research on hiring discrimination towards sexual minorities is, amongst others, done by Baert (2014, 2018b), Drydakis (2009, 2014, 2015), Van Hoye and Lievens (2003), and Weichselbaumer (2015).

4 'Transphobia' is defined by Hill and Willoughby (2005) as an emotional disgust relative to people who do not meet social expectations about gender.
} 
labour market outcomes for transgender people. Another negative stereotype, regarding transgender men in particular, is the fact that, because transgender men were assigned the female sex at birth, it is possible they are ascribed less typically male characteristics associated with labour market success, such as assertiveness and autonomy (Padavic and Reskin, 2002; Schilt and Wiswall, 2008), and as a consequence are perceived as less productive compared with cisgender men. The negative perception regarding their autonomy and assertiveness might, therefore, lead to more negative labour market outcomes for them (Padavic and Reskin, 2002; Schilt and Wiswall, 2008). Lastly, both transgender men and women may be perceived as having a lower probability to fall out of the labour market because of parental leave, since they are assumed to have fewer children than cisgender men and women, and therefore, might avoid the well-documented penalty of parenthood in the labour market (Baert, 2014; Baert et al., 2019; Van Borm and Baert, 2018). ${ }^{5}$

Although previous theoretical literature has mentioned several potential explanations for the unequal treatment between cisgender and transgender persons in the labour market, as discussed above, research that investigates these theoretical explanations empirically is, however, scarce. To the best of our knowledge, there have been only two studies that empirically explore potential explanations for labour market discrimination towards transgender individuals, i.e. Reed et al., (2015) and Van Borm and Baert (2018). First, Reed et al. (2015) explored the mediating role of the abovementioned negative stereotype about transgender individuals' perceived health status on their hiring chances. To this end, they used a scenario experiment in which American participants had to rate one out of four hypothetical profiles, varying in gender identity (i.e. transgender or cisgender) and sex (i.e. male or female), with respect to their perceived health and probability to get hired. The authors found that the transgender candidates, indeed, were perceived as being in worse health than cisgender candidates and that this perception, in turn, had a negative effect on the hiring chances of the candidates. In addition, the researchers found that the mental health perceptions robustly explained the adverse hiring outcomes for transgender men, but less strongly for transgender women.

Second, Van Borm and Baert (2018) explored all potential explanations for discrimination towards transgender women, discussed above, in the hiring context. To this end, the researchers conducted a scenario experiment similar to the one of Reed et al. (2015), in which Belgian final-year students evaluated a fictive profile of a transgender or a cisgender woman regarding (i) the probability with which they would invite the candidate to a job interview, (ii) the probability with which they would hire the candidate, and (iii) seven statements related to the economic theories of taste-based and statistical discrimination. Although participants indicated to feel comfortable with collaborating with transgender women themselves, they also indicated to suppose that their customers and other employees would have an aversion for collaborating with transgender women. Next to this, the participants indeed perceived transgender women to be in worse health, to be more autonomous and assertive, and to have a lower probability to go on parental leave compared with their cisgender counterparts. Of these different attitudes and

\footnotetext{
5 This idea might be related to the irreversible sterilisation that transgender people undergo in certain medical procedures (De Sutter, 2001).
} 
stereotypes, the perception about transwomen's health, autonomy and assertiveness acted as dominant explanations for variation in their hiring probability.

Next to the different explanations for hiring discrimination towards transgender women, Van Borm and Baert (2018) also explored the heterogeneity in unequal treatment between transgender and cisgender women by different participant and job characteristics. With respect to participant characteristics, the authors, for example, argued that men might discriminate more towards transgender women compared with cisgender women because previous research has found cisgender men to be more transphobic than cisgender women (Nagoshi et al., 2008; Norton and Herek, 2013). In addition, with respect to job characteristics, Van Borm and Baert (2018) expected the degree of discrimination towards transgender women to differ in jobs with different gender types (i.e. male-dominated, gender-neutral, and female-dominated jobs; Heilman, 1983; Van Borm and Baert, 2018). They, however, did not find significant moderation effects of (i) participant's gender, (ii) the degree to which participants believe in classical gender roles, (iii) the amount of contact participants have with transgender persons, (iv) participants' sexual orientation, and (v) the gender type of a job on the degree of hiring discrimination towards transgender women.

Although the studies of Reed et al. (2015) and Van Borm and Baert (2018) are innovative, since they are the first to explore different explanations for hiring discrimination towards transgender individuals, they are both limited in at least one aspect. On the one hand, the research of Reed et al. (2015) has three main limitations. First, the researchers only consider the health of transgender men and women as a potential explanation for hiring discrimination towards them. As mentioned above, there are, however, other potential mechanisms underlying the unequal treatment between cisgender and transgender people (transgender men in particular), such as the perception about their autonomy and assertiveness. Second, although the authors survey their participants with respect to several demographic characteristics, such as their gender, they do not explore the heterogeneity in unequal treatment between cisgender and transgender individuals by these characteristics. There are, however, as mentioned above, reasons to believe that the degree of discrimination could differ by these characteristics. Third, Reed et al. (2015) take into account only one particular position (i.e. Radiologic Technician) in their experimental design. This has two main implications: (i) it makes it impossible to explore the heterogeneity in hiring discrimination towards transgender individuals by types of jobs and (ii) it limits the external validity of their research considerably. Their results can, therefore, not be generalised to other contexts.

On the other hand, although Van Borm and Baert (2018) do take into account several potential explanations (i.e. taste-based and statistical discrimination) and moderators (i.e. participant and job characteristics) in their research, in contrast to Reed et al. (2015), their research is limited, because it only focusses on hiring discrimination towards transgender women. There are, however, two main reasons for why it is necessary to research hiring discrimination towards transgender men as well. First, it provides us with a more complete view on the potential explanations of hiring discrimination towards transgender individuals and enables us to compare the results regarding transgender women with those on transgender men. Indeed, there are several reasons to believe that the explanations for hiring discrimination towards 
transgender men and transgender women may differ. More concretely, transphobia towards transgender men is expected to be lower than against transgender women partly because transgender men usually pass more easily as a member of the opposite gender (Bockting et al., 2013; Schilt and Wiswall, 2008; Worthen, 2013). ${ }^{6}$ As a consequence, we may expect transphobia to be a less important explanation for hiring discrimination towards transgender men than it is for hiring discrimination towards transgender women. In addition, transgender men's health is supposed to differ from that of transgender women. More precisely, transgender men are associated with lower levels of mental health issues (and suicide) and with lower risks at contracting HIV than transgender women (Bockting et al., 2013; Clements-Nolle et al., 2006; Drydakis, 2017a; Hughto et al., 2015). We could thus expect that the health-related stigma would play a smaller role in explaining hiring discrimination towards transgender men compared with transgender women. ${ }^{7}$ Next to transphobia and perceived health, transgender men and women might also differ in terms of autonomy and assertiveness. It is possible that transgender men are perceived as more autonomous and assertive than transgender women because they are assumed to be more masculine and as a result are associated with higher levels of labour market success (Baert, 2014; Gorsuch, 2019; Schilt and Wiswall, 2008; Van Borm and Baert, 2018).

Second, it is necessary to conduct research on the explanations of hiring discrimination towards transgender men because of the simple fact that hiring discrimination towards transgender men, even though less severe than against transgender women ${ }^{8}$ (Grant et al., 2011), is a serious problem that should be addressed (FRA, 2014; Grant et al., 2011; Rainey et al., 2015). To develop adequate policy actions to tackle this issue, it is important to get deeper insights into why employers discriminate towards transgender men (mediators) and in which situations this discrimination is higher (moderators).

In this study, we, therefore, investigate the same potential mechanisms and moderators of hiring discrimination towards transgender women as Van Borm and Baert (2018) applied to transgender men. That is, we explore whether (i) transphobia, (ii) the perception of transgender men's health, (iii) the perception of transgender men's autonomy and assertiveness, and (iv) transgender men's perceived parental leave probability drives hiring discrimination towards transgender men. In addition, as Van Borm and Baert (2018), we examine the heterogeneity in unequal treatment between cisgender and transgender men by several participant and job characteristics. We, however, extend the set of moderators of Van Borm and Baert (2018) with

\footnotetext{
${ }^{6}$ This is because of the differential impact of masculinising versus feminising hormones (Bockting et al., 2013; Schilt and Wiswall, 2008; Worthen, 2013).

${ }^{7}$ However, as mentioned before, Reed et al. (2015) found that mental health perceptions robustly explained the adverse hiring outcomes for transgender men, but less strongly for transgender female applicants.

${ }^{8}$ Previous research has found that women who do not behave conform their gender at birth (e.g. transgender men) are usually penalised less than gender non-conforming men (e.g. transgender women; Blakemore, 2003; Levy et al., 1995; Zucker et al., 1995) because of the lower level of flexibility men have with respect to their gender expression (Bockting et al., 2013; Worthen, 2013). This lower level of flexibility might be established through the prevalence of patriarchy in many of the western societies and women's emancipation. Where, throughout history, men have guarded their superior position installed through the typical male gender roles (i.e. hegemonic masculinity; Bird, 1996), women have combatted the typical female gender roles to overcome their inferior position with respect to men. As a consequence, these typical female gender roles have become more flexible, as well as women's gender expression, as opposed to the one's of men.
} 
one additional participant characteristic: the degree to which recruiters are risk-averse. We decided to include this extra moderator based on the findings of Baert (2018b), who found that homosexual men face higher levels of hiring discrimination when employers are more riskaverse. To examine these different explanations and moderators of hiring discrimination towards transgender men, we mimic the scenario experiment of Van Borm and Baert (2018), also, using final-year students as participants.

The main aim of this study is, therefore, not to measure hiring discrimination towards transgender men, as done by Bardales (2013), Granberg et al. (2019), Make The Road New York (2010), and Rainey et al. (2015) using audit and correspondence tests, but to explain this discrimination using a scenario experiment. We contribute to the academic literature by exploring four potential explanations for hiring discrimination towards transgender men, as well as six different moderators of this discrimination, therefore supplementing the research of Reed et al. (2015) and Van Borm and Baert (2018), who, respectively, only investigated the health-related stigma of transgender individuals as potential explanation for hiring discrimination towards transgender individuals or focused on transgender women only. In doing so, we are able to compare the results regarding transgender women with those of transgender men and get a more complete view on the issue of hiring discrimination towards transgender people. More concretely, we are able to get deeper insights into why employers discriminate (mediators) towards transgender individuals and in which situations this discrimination is higher (moderators), which is necessary information to develop adequate policy actions and combat hiring discrimination towards them.

\section{Method}

As mentioned before, we mimicked the scenario experiment of Van Borm and Baert (2018) to get deeper insights into why employers potentially discriminate towards transgender men in the hiring process. Both the data collection process (Subsection 2.1), as well as the analysis strategy to analyse the experimentally gathered data (Subsection 2.2) are discussed in the present section.

\subsection{Data}

\subsubsection{Participant selection}

We conducted the experiment in October 2018 with students enrolled at Ghent University, the second biggest university of Flanders (Belgium). To participate in the experiment, the students had to meet two criteria. First, they had to be final-year students. Second, they had to be registered in a master's degree programme related to Human Resource Management. We selected the students based on these two criteria because we believe that final-year students enrolled in study programmes related to Human Resource Management have the necessary background knowledge to make rational and well-thought hiring decisions. 
To reach these students, we first listed all eligible study programmes using the available course catalogue on the website of Ghent University, a tool that provides information about the study programmes and available courses. In total, we selected 16 programmes taught at Ghent University that related to Human Resource Management. Next, we contacted all 16 lecturers who instructed a course in one of these study programmes and asked them whether we could conduct our experiment in their class. Five of them agreed to this. In the end, 252 students, enrolled in five different study programmes, participated in this experiment.

\subsubsection{Experiment}

Like Van Borm and Baert (2018), we conducted our experiment by means of a paper-and-pencil survey comprising two booklets: one containing experimental instructions and one containing a post-experimental questionnaire. Both booklets were adopted from Van Borm and Baert (2018) and adjusted to a limited extent to match our research goals. The participants were instructed to first fill out the booklet containing experimental instructions. After the experiment, they had to fill out the post-experimental questionnaire, without returning to the former booklet.

The booklet with experimental instructions (hereafter 'the vignette') consisted of three elements: (i) a hypothetical vacancy, (ii) a fictitious resume, and (iii) an evaluation form. At the beginning of the vignette, the participants were instructed to take on the role of a recruiter for a firm selling building materials who had to fill a vacancy for one of three occupations, which varied with respect to gender type. More concretely, they had to fill the job of management assistant (female-dominated job), logistics clerk (gender-neutral job), or site manager at a building yard (male-dominated job). These jobs (and the associated job descriptions) were, again, adopted from Van Borm and Baert (2018) who selected them based on numbers of the Public Employment Agency of Flanders (PEAF) concerning the gender representation in these occupations. ${ }^{9}$ Descriptions of the different jobs and the required profiles can be found in Table A1 in the Appendix.

After reading the instructions, the students had to screen one vacancy and associated resume of a male applicant with the required qualifications for the job. This male applicant held a bachelor's degree corresponding to the one required in the vacancy and had five years of experience in the same occupation for which he applied. The resume also contained the date and place of birth, nationality, and marital status of the applicant. Besides this, the applicant was skilled in four languages - Dutch (excellent), English (very good), French (very good) and German (good) — and had excellent computer skills (office applications). ${ }^{10}$ The resume also revealed five personal characteristics: stress-proof, organised, a team player, communicative and punctual.

Next to diversifying between the three jobs, we randomly alternated the gender identity of the applicant (i.e. trans or cis identity). Following Van Borm and Baert (2018), we revealed the applicant's gender identity by means of two indicators. ${ }^{11}$ First, the name of the applicant was mentioned. On the cisgender profile, only the typical Flemish male name 'Tom Mertens' was

\footnotetext{
${ }^{9}$ Management assistant: $8.1 \%$ male; Logistics clerk: 52.6\% male; Site manager: $86.2 \%$ male.

${ }^{10}$ Belgium has three official languages: Dutch, French and German. Being skilled in Dutch, French, and German, is therefore not exceptional in Belgium.

${ }^{11}$ A more elaborated discussion on the choice for these two indicators can be found in Van Borm and Baert (2018).
} 
mentioned. On the transgender profile, however, the same Flemish name was mentioned and supplemented with a subtitle that stated the candidate was born as 'Sarah Mertens'. ${ }^{12}$ Second, the gender of the applicant was mentioned. Again, on the cisgender profile, only the word 'male' was mentioned, whereas on the transgender profile the word 'transgender' was mentioned between brackets in addition to the word 'male'. The remark that has to be made here is that, as a consequence of our design, the transgender candidate in our experiment is, in fact, an openly transgender candidate and the cisgender candidate is actually a candidate with an unrevealed status in this respect. We will return to this in Section 4.

After screening the fictitious vacancy and resume, the students had to evaluate their candidate concerning two clusters of items. First, they had to indicate their general intention to hire the job candidate. More specifically, in line with Baert (2018), Van Borm and Baert (2018), and Van Hoye and Lievens (2003), they had to rate one statement concerning the probability with which they would invite the candidate to a job interview (i.e. 'I will invite this candidate for a job interview') and one concerning the probability with which they would actually hire the candidate (i.e. 'I will hire this candidate'), both on a 7-point Likert scale ranging from 'very unlikely' (score 1) to 'very likely' (score 7). In what follows, we will refer to these scores as the 'interview probability' and 'hiring probability' of the candidates, respectively.

Next and more importantly, the students had to rate seven statements related to the potential explanations for hiring discrimination against transgender men (i.e. taste-based discrimination and statistical discrimination). To measure potential taste-based discrimination, the participants had to rate three statements related to employer discrimination, co-worker discrimination and customer discrimination, in line with Baert and De Pauw (2014) and Van Borm and Baert (2018): (i) 'As an employer, I will enjoy collaborating with this candidate' (employer discrimination), (ii) 'My co-workers will enjoy collaborating with this candidate' (employee discrimination), and (iii) 'My customers will enjoy collaborating with this candidate' (customer discrimination). To measure statistical discrimination, rooted in productivity-related prejudices on the health, autonomy, assertiveness and likelihood of paternity leave of a job candidate, the following statements were presented, again in line with Van Borm and Baert (2018): (i) 'This candidate will be on sick leave in the short or medium run', (ii) 'This candidate will provide sufficient autonomy', (iii) 'This candidate will provide sufficient assertiveness', and (iv) 'This candidate will be absent in the short or medium run because of having children'. All seven statements had to be scored on a 7-point Likert scale ranging from 'completely disagree' (score 1) to 'completely agree' (score 7). In what follows, we refer to these seven statements as the different 'candidate evaluation scales'.

\subsubsection{Post-experimental questionnaire}

As mentioned, the students had to fill out a post-experimental questionnaire after they filled out the vignette. This post-experimental survey was the same for each participant, regardless of the

\footnotetext{
${ }^{12} \mathrm{We}$ are aware that the wording used could be perceived as insensitive, however, to assure it was easy to understand for the participants what was meant, we decided to formulate the subtitle in the abovementioned direct and clear way.
} 
vignette they evaluated. At the beginning of the post-experimental questionnaire, it was mentioned that the students could leave their role of recruiter and that they had to fill out the survey from a personal point of view.

The post-experimental questionnaire consisted of six different elements. First, the participants had to answer a manipulation check containing four statements concerning the identity of the candidate, more specifically, his gender, residence, ethnic origin and whether the candidate applied for a job in which women were underrepresented. Again, these statements were scored on a 7-point Likert scale ranging from 'completely disagree' (score 1) to 'completely agree' (score 7). The statement regarding the gender of the applicant was included to verify whether it was clear to the respondents that they had assessed a trans or cisgender candidate and thus, whether the experimental manipulation was successful. To avoid emphasising the goal of the research too much, the three other (redundant) statements concerning the candidate were added.

Second, the participants' social desirability bias was measured using the 13-item version of the Marlowe-Crowne Social Desirability Scale (MC-SDS) developed by Reynolds (1982), one of the most used instruments for measuring social desirability (Baert, 2018b, Beretvas et al., 2002; Sârbescu et al., 2011). This scale consists of 13 items concerning behaviour that is perceived as desirable or undesirable within society, such as 'There are moments where I took advantage of someone' and 'There have been moments where I was jealous of someone's happiness'. The participants were asked to indicate whether they agreed or disagreed with these different propositions by means of a true or false question. Socially desirable answers were coded as 1 and non-socially desirable answers as 0 . To obtain a total score for social desirability, scores given on each statement were summed up, resulting in a score between 0 and 13 . This score was then divided by 13 to get a number between 0 and 1, which represented the participants' score on the social desirability scale. Scores closer to one are associated with high levels of social desirability. For our sample of participants, the Cronbach's alpha coefficient of this scale is 0.521 .

Third, the degree to which the participants are risk-averse was measured by means of two subscales of three items selected from the Domain-Specific Risk-Taking (DOSPERT) Scale of Blais and Weber (2006), in analogy with Baert (2018b). The first sub-scale measured 'social risks', and the second sub-scale measured 'financial risk preferences'. We decided to include only these two sub-scales of the DOSPERT Scale because, given the labour market context of our study, these two sub-scales were perceived as the most relevant. The participants had to indicate the probability in which they would take part in six different specific activities on six 7-point Likert scales ranging from 'very unlikely' (score 1) to 'very likely' (score 7). Examples of the items are: 'investing $10 \%$ of your yearly income in a new company' and 'disagreeing with a superior (for example your boss) on an important issue'. We averaged the scores on each statement, to obtain a total score for risk aversion, where a higher score is associated with a 
high level of risk aversion. ${ }^{13}$ The Cronbach's alpha coefficient of this scale is 0.431 , for our specific sample.

Fourth, the participants' belief in traditional gender roles was surveyed. This was done by means of a shortened version of the Attitudes Toward Women Scale (AWS) of Spence et al., (1973). The participants had to rate, again on a 7-point Likert scale ranging from 'completely disagree' (score 1) to 'completely agree' (score 7), to what extent they agreed with 24 statements concerning the desirable roles and rights of women in different contexts. Examples of these statements are: 'Swear words expressed by women are more repulsive than swear words expressed by men' and 'Women should have the same privileges with regard to promotion and recruitment as men'. Averaging the score of these statements yielded a number between 1 and 7 on the conformity to classical gender roles scale, where a higher score is associated with high conformity to classical gender roles. For our sample of participants, the Cronbach's alpha coefficient of this scale is 0.867 .

The fifth element related to the participants' contact with transgender individuals. More concretely, we used the scale of West and Hewston (2012), which consists of four items, to examine the amount of contact participants had with transgender people in four different contexts: (i) at work or at school, (ii) in daily superficial social situations, (iii) in intimate social situations, and (iv) in all kinds of social situations. For each item the participants had to score their level of contact with transgender individuals, ranging from 'no contact at all' (score 1) to 'a lot of contact' (score 7). By taking the average score of these statements we got a number between 1 and 7 ('contact with transgender scale'), where a score closer to seven was associated with a higher degree of contact with transgender individuals. The Cronbach's alpha coefficient of this scale is 0.720 .

Finally, the social background of the students was surveyed by means of seven items: their gender (female, male or x), age, field of study, nationality (Belgian, non-Belgian but EU-27 or non-EU-27), the highest education degree of their father (tertiary education, secondary education or lower), the highest education degree of their mother (tertiary education, secondary education or lower), and sexual orientation (heterosexual, bisexual or homosexual).

\subsubsection{Data description}

In Table 1 we present summary statistics regarding the manipulation check (Panel A) and the occupation and participant characteristics (Panels B and C) for the two experimental conditions (i.e. the subsamples of participants classified by the gender identity of their assigned job candidate). We ran two-tailed t-tests to test whether the differences in ratings between the

\footnotetext{
${ }^{13}$ We recoded the scores ranging from 1 'very likely' to 7 'very unlikely' to have a more logical coding for the risk aversion scale, where high scores on the scale resemble risk-averse participants and low scores risk seeking participants.
} 
experimental group (i.e. transgender profiles) and the control group (i.e. cisgender profiles) are significantly different from 0 . Significance is marked at the $10 \%$ level. ${ }^{14}$

\section{< TABLE 1 ABOUT HERE >}

As shown in Panel A, our experimental manipulation worked. The average rating on the statement 'The candidate was born as a woman'" ${ }^{15}$ is 6.532 among the students who were allocated a transgender candidate, while the average rating is only 1.500 among those allocated a cisgender candidate. The difference between these scores is highly significant $(p=0.000)$. In other words, the participants were very much aware of the gender identity of the applicant they had to evaluate.

Next, as becomes clear in Panel B, the randomisation of the three types of vacancies over the participants (in the two experimental conditions) was successful as well. The six potential combinations of the three occupations and two transgender identities are evenly represented in the sample. In addition, the randomisation of the transgender identity over the participants of the experiment also succeeded (Panel C). Consequently, the participants in both experimental conditions are very similar in terms of social background, risk aversion, conformity to classical gender roles, contact with transgender individuals, and social desirability bias. ${ }^{16}$

\subsection{Analyses: Statistical framework}

Before discussing our results (infra Section 3), we first describe the statistical framework we use to analyse this data in the present subsection.

To answer our two research questions (i.e. (i) why do employers discriminate (mediators) and (ii) in which circumstances is this discrimination higher (moderators)?) we proceed in three different steps: (i) we explore the explanations of hiring discrimination towards transgender men by means of a bivariate analysis (infra Subsection 3.2), (ii) we investigate these explanations in more detail by running a multiple mediation model in which all explanations are included jointly (infra Subsection 3.3), and (iii) we research potential moderation effects of different participant and job characteristics by means of a moderation analysis (infra Subsection 3.4). Before answering our two research questions, however, we briefly discuss the total effect of one's gender identity on hiring chances (i.e. interview and hiring probability; infra Subsection 3.1). It has to be highlighted here that identifying the effect of one's transgender status on hiring chances is not the main goal of our research since a vignette experiment is a

\footnotetext{
${ }^{14}$ We chose to mark significance at the $10 \%$ level based on several other studies, which also marked the significance at the 10\% level (e.g. Van Belle et al., 2018; Van Borm and Baert, 2018). By working with several significance levels and presenting the t-statistics, we allow the reader to interpret the results themselves.

${ }^{15} \mathrm{We}$ are aware that the wording used could be perceived as insensitive, however, to assure it was easy to understand for the participants what was meant, we decided to formulate the manipulation check in the abovementioned direct and clear way.

${ }^{16}$ From Table 1 (Panel C) it can be seen that the subsample of students who were allocated a transgender profile seemed to consist of more people with a non-Belgian nationality than the subsample of participants who were allocated a cisgender profile. The difference between the two groups concerning nationality is statistically significant. The difference is only weakly significant at the 5\% level, so the occurrence of a Type-I error can, therefore, not be ruled out.
} 
less appropriate method to measure hiring discrimination towards transgender men compared with audit and correspondence studies. However, we believe it is still interesting to briefly explore the effect to investigate whether our vignette experiment can replicate the findings of earlier correspondence experiments.

As mentioned above, we start Section 3 with a brief discussion of the total effect of one's transgender status on their hiring chances (i.e. interview and hiring probability; infra Subsection 3.1). More concretely, we examined whether participant's ratings of the interview probability scale and hiring probability scale differ by the gender identity of the job candidate they had to rate. To this end, we ran two-tailed t-tests to test whether the differences in ratings on both scales between the cisgender and transgender candidates significantly differ from zero. We marked the significance at the $10 \%$ level for the same reason as we did when describing the data in Subsection 2.1.4 (supra footnote 16).

Next, with respect to our first research question, we ran a bivariate analysis to explore the potential explanations for hiring discrimination towards transgender men. This analysis consists of two main parts. More concretely, we first examined whether one's gender identity has an effect on the different candidate evaluation scales related to the theories of taste-based and statistical discrimination (discussed in Subsection 2.1.2), and thus examined what one's gender identity signals to employers. To this end, we, again, ran two-tailed t-tests to test whether the differences in ratings on the different candidate evaluation scales between the cisgender and transgender candidates significantly differ from zero and mark the significance at the $10 \%$ level. Subsequently, we then investigated whether these candidate evaluation scales are associated with the hiring decisions made (i.e. a candidate's interview and hiring probability). Since we did not experimentally manipulate the different signals of one's transgender status, we can only speak of associations between the candidate evaluation scales and the outcome variables and not of causal effects. We, therefore, calculate the correlation coefficients between the different candidate evaluation scales and the interview and hiring probability.

Based on the bivariate analysis of our results, we, however, could not be sure whether the relationships found indeed existed or whether they were spurious associations, picking up other, genuine relations. Therefore, we subsequently ran a multiple mediation model in which all potential explanations were included jointly to identify the dominant explanations for hiring discrimination towards transgender men. We discuss this multiple mediation model in more detail in Subsection 3.3.

Last, with respect to our second research question, we divided our total sample into different subsamples based on different participant and job characteristics to identify potential moderation effects of these different characteristics on the degree of discrimination towards transgender men. In particular, we tested whether the ratings of the hiring probability scale between transgender and cisgender job applicants were significantly different from zero in these different subsamples, again, by means of two-tailed t-tests (infra Subsection 3.4). Significance was again marked at the $10 \%$ level. Additionally, we ran a multivariate regression analysis with interaction terms to investigate the potential moderation effects of the different job and participant characteristics in another way. Newsom et al. (2003) and Stone-Romero and Anderson (1994) argue that using regressions with interaction terms to test for variation by subgroup is recommended because the approach would lead to fewer erroneous conclusions than when dividing the sample by group and run separate analyses on each of the subgroups. 


\section{Results}

In this section, we discuss the results obtained through the statistical analyses discussed above (supra Subsection 2.2).

\subsection{The total effect of one's transgender status on hiring chances}

As discussed above (supra Subsection 2.2), we first briefly explore what the effect is of revealing a transgender status on a resume on a job candidate's hiring chances using two-tailed $\mathrm{t}$-tests. The results of these t-tests are presented in Table 2 (Panel A).

\section{< TABLE 2 ABOUT HERE >}

As becomes clear from Table 2 (Panel A), we find no effect of one's transgender identity on both interview probability and hiring probability in our experiment. This non-negative effect is in contrast with the findings of Bardales (2013), Make The Road New York (2010), and Rainey et al. (2015), who all found a significant negative effect of one's transgender identity on hiring chances in the United States, and those of Granberg et al. (2019) who found similar results in Sweden.

There are three potential reasons for why we do not find a similar negative effect in our experiment. First, our vignette experiment was conducted in Belgium, i.e. a country with a rather tolerant public opinion towards sexual and gender minorities (Baert, 2018b; ILGAEurope, 2019; OECD, 2019). ${ }^{17}$ Second, because we use students instead of real recruiters, our sample mainly consists of young people. Young individuals are found to be more open-minded and tolerant towards transgender individuals than older individuals (King et al., 2009; Landén and Innala, 2000) which could explain the non-negative effect found in our research. The last potential explanation for the abovementioned result can be linked to the limitations related to our research method - the vignette experiment. ${ }^{18}$ One of the disadvantages of working with a vignette experiment is the experimental setting. Because our participants are students making fictitious hiring decisions, they do not face the consequences of their decision, which could make them behave differently from when they would make real-life hiring decisions. Additionally, because participants know they are partaking in an experiment, they may answer in a more socially desirable way. To control for this social desirability bias, in Table A2 in the Appendix, we provide the candidates' evaluation scores after excluding those participants with a score for the social desirability bias scale higher than the total sample mean increased with one standard deviation (Panel A) and a social desirability score higher than the total sample

\footnotetext{
17 The idea that Belgium is a tolerant country is confirmed by the results of previous research studying hiring discrimination towards LGBT-individuals in Belgium. Baert (2014, 2018b) and Van Borm and Baert (2018) all found a non-negative effect of one's homosexuality or transgender status on hiring chances.

${ }^{18} \mathrm{We}$ will come back to the limitations related to the usage of a vignette experiment in Section 4.
} 
mean (Panel B). We, however, obtain very similar (non-negative) results after excluding those participants from the sample with a high tendency to answer in a socially desirable matter. Our results are, therefore, robust for social desirability. Although we did not find evidence for hiring discrimination towards transgender men within the context of our experiment, it is still important to investigate the mechanisms underlying this result and get deeper insights into why we find this result. In the following two subsections, we, therefore answer our main research question by focussing on the different explanations for hiring discrimination towards transgender individuals related to the theories of taste-based and statistical discrimination.

3.2. Explanations for hiring discrimination against transgender men: Bivariate analysis

In this subsection, we explore the different explanations of hiring discrimination towards transgender men separately. As discussed in Subsection 2.2, our statistical analysis consists of two main analyses (i.e. running two-tailed t-tests and calculating correlation coefficients).

With respect to the first analysis, we focus on the effect of one's gender identity on the different candidate evaluation scales related to the theoretical models of taste-based and statistical discrimination, outlined in Subsection 2.1.2, to find out what exactly is being signalled by one's transgender status. The results of the t-tests are presented in Table 2 (Panel B).

As shown in Table 2 (Panel B), we find significant effects of one's gender identity on all different candidate evaluation scales, except for one. More concretely, the average score on the statement 'As an employer, I will enjoy collaborating with this candidate' does not significantly differ between the experimental group (i.e. the group of students that received a resume of a transgender candidate) and the control group (i.e. the group of students that received a resume of a cisgender candidate). In other words, the participants in our experiment do not seem to prefer working with a cisgender employee compared with a transgender employee. We thus find no evidence for employer discrimination towards transgender men.

Moreover, although we do not find any evidence for employer discrimination, we do find suggestive evidence for co-worker and customer discrimination. The average score for the cisgender candidate is indeed significantly higher than the score for the transgender candidate (i.e. 5.000 versus 4.412) on the statement 'The other employees will enjoy collaborating with this candidate', as well as on the statement 'Customers will enjoy collaborating with this candidate' (i.e. 4.889 versus 4.440). It thus seems the participants in our experiment would feel comfortable working with a transgender employee; however, it also seems they suppose their co-workers and customers would not.

In addition, based on the literature discussed in Section 1, we expected that transgender men would be perceived to be in worse health than cisgender men. The results in Table 2 (Panel B) confirm these expectations: the average score for the transgender candidate is indeed significantly higher than the average score for the cisgender candidate (i.e. 2.700 versus 3.262). 
Concerning the perceived assertiveness and autonomy of transgender men, we expected that transgender men would be perceived as less autonomous and assertive than cisgender men because they were assigned the female sex at birth (see Section 1; Baert, 2014; Schilt and Wiswall, 2008). The results presented in Table 2 (Panel B) show the opposite: the average score for the transgender candidate for perceived autonomy (i.e. 4.944 versus 5.413) and perceived assertiveness (i.e. 4.841 versus 5.238) are significantly higher than the corresponding average scores for the cisgender candidate. A potential explanation for these results is the idea that transgender individuals might act in a more autonomous and assertive way as a reaction to the stigma linked to their transgender status. In other words, transgender individuals might use autonomy and assertiveness as a stigma management tool to deal with the stigma they experience in their day-to-day lives, which might lead to the fact that the participants in our experiment, indeed, perceived transgender individuals as more assertive and autonomous (Bockting, 2014).

Last, looking at the effect of one's transgender status on perceived paternity leave probability, cisgender men are indeed perceived as more likely to go on paternity leave than transgender men. As shown in Table 2 (Panel B), the average score for the cisgender candidate is significantly higher than the average score for the transgender candidate (i.e. 2.944 versus 2.444). This is in line with what we expected based on the literature.

With respect to the second statistical analysis, we investigate, as mentioned in Subsection 2.2, the association between the abovementioned candidate evaluation scales and the candidates' interview and hiring probability by calculating correlation coefficients. The correlation matrix can be found in Table 3 .

\section{< TABLE 3 ABOUT HERE >}

We find, as expected, that a more positive perception concerning personal taste, co-workers' taste and customers' taste for collaboration with a candidate is significantly related to a higher interview and hiring probability. Further, we find a highly significant correlation between the perceived autonomy and assertiveness of a candidate and the two outcome variables. We, however, do not find a significant correlation between the perceived parental leave probability and both outcome variables, nor do we find a significant correlation between the perceived health status of a candidate and his interview probability. We do find a weakly significant (only at the $10 \%$ level) correlation between the perceived health status of a candidate and his hiring probability.

Taking the two steps of our statistical analysis together, we can conclude that a bivariate analysis of our data points to the direction of a considerable negative mediating role for (i) the fear of participants that co-workers and customers would rather work with cisgender men than with transgender men and, to a lesser extent, (ii) the health-related stigma (only for hiring probability). In addition, we find suggestive evidence of a significant positive mediating role for (i) perceived autonomy and (ii) perceived assertiveness. Based on our bivariate analysis of our data, we thus find results similar to Van Borm and Baert (2018). 
As Columns (3) to (9) of Table 3 show, the different candidate evaluation scores are highly correlated. As a consequence, it can be the case that the suggested mediation roles are nothing more than spurious associations picking up other relations (supra Subsection 2.2). To control for this issue, we run, as mentioned, a multiple mediation model, in which we include all potential explanation jointly, to see whether the mediation effects are indeed independent. We discuss this multiple mediation model in more detail in the next section.

\subsection{Explanations for hiring discrimination against transgender men: Multiple mediation model}

In Figure 1, we present a mediation model in which the direct effect of one's transgender status on the outcome variable 'hiring probability' is explained by the different mediators discussed in Subsection 2.1.2 and 3.2 by decomposing the direct effect into a direct association and different indirect associations via the different mediators. Following Van Borm and Baert, (2018), we used the averaged mean values of the statements related to co-worker and customer discrimination as one 'co-worker and customer taste to collaborate' scale (Cronbach's $\alpha$ is 0.818 ) and the averaged mean values of the statements related to perceived autonomy and assertiveness as one 'perceived autonomy and assertiveness' scale (Cronbach's $\alpha$ is 0.731) in the mediation model. ${ }^{19}$ In analogy with the aforementioned study, the three other evaluation scores (i.e. employers' taste to collaborate, perceived paternity leave probability, and perceived sick leave probability) were included in the model as separate mediators. The resulting mediation model, therefore, consists of a system of six linear regression equations. In the first five regression models, the mediators are regressed on a dummy concerning the job candidate's transgender status (taking on the value 1 when it concerns a transgender profile and taking on the value 0 when it concerns a cisgender profile). In the last regression model, the outcome variable 'hiring probability' is regressed on the five mediators and the transgender dummy. All included variables, with exception of the transgender dummy, are standardised by subtracting their sample mean and dividing the results by their sample standard deviation. Like Van Borm and Baert (2018), we estimated the six regression models following the procedure discussed in Hayes (2013). The estimation results of these six regression models can be found in Figure 1.

\section{< FIGURE 1 ABOUT HERE >}

Within the context of our experiment, the total effect of one's transgender status on hiring probability is, indeed, as discussed in Subsection 3.1, not statistically significant $(c=0.081, p$ $=0.126)$. As mentioned, this effect can be decomposed into a direct effect and different indirect associations through the mediators (i.e. mediation effects), where the mediation effects are the products of the effect of the transgender dummy on the relevant mediator (i.e. the $a_{i}$ in Figure 1 ) and the association of this mediator with the hiring probability (i.e. the $b_{i}$ in Figure 1 ).

\footnotetext{
19 Van Borm and Baert (2018) grouped the four different statements into two clusters because of the high correlation between the statements related to co-worker and customer discrimination, on the one hand, and the high correlation between the statements related to perceived autonomy and assertiveness, on the other hand.
} 
First, looking at the direct association between a candidate's transgender status and hiring probability, we can see that the model with the five mediators included jointly, to a large extent explains the total effect of one's transgender identity on hiring probability. The remaining direct association between the transgender status of a candidate and the hiring probability, after controlling for the five included mediators, is not significant $\left(c^{\prime}=0.109, p=0.112\right)$.

Next, focusing on the effect of the transgender dummy on the different mediators (i.e. the lefthand side $a_{i}$ of Figure 1), we find a statistically significant negative effect of the transgender dummy on (i) co-workers' and customers' taste to collaborate with a candidate $\left(a_{2}=-0.526, p\right.$ $=0.121)$ and (ii) the perceived paternity leave probability of a candidate $\left(a_{4}=-0.377, p=\right.$ $0.124)$, a statistically significant positive effect of the transgender dummy on (i) the perceived autonomy and assertiveness $\left(a_{3}=0.460, p=0.123\right)$ and (ii) the perceived sick leave probability of a candidate ( $a_{5}=0.412, p=0.123$ ), and no effect of the transgender dummy on the participants' taste to collaborate with a candidate $\left(a_{1}=-0.047 p=0.126\right)$. These findings are, not surprisingly (given the random allocation of the transgender and cisgender profiles to the participants), completely in line with the statistics presented in Table 2 (Panel B).

Furthermore, when looking at the association of the mediators with the hiring probability (i.e. the right-hand side $b_{i}$ of Figure 1) we find that only (i) the participants' taste to collaborate ( $b_{1}$ $=0.211, p=0.066)$, (ii) co-workers' and customers' taste to collaborate $\left(b_{2}=0.231, p=0.069\right)$, and (iii) high perceived autonomy and assertiveness $\left(b_{3}=0.287, p=0.071\right)$ are significant (positive) drivers of hiring probability when controlling for all mediators jointly, while perceived paternity leave probability $\left(b_{4}=-0.005, p=0.058\right)$ and perceived sick leave probability $\left(b_{s}=-0.076, p=0.056\right)$ are not.

Multiplying the estimated $a_{i}$ and $b_{i}$ coefficients for each mediator yields two statistically significant mediation effects. More concretely, we find a highly significant negative mediation via co-workers' and customers' taste to collaborate $\left(a_{2} b_{2}=-0.121, p=0.047\right)$ and a highly significant positive mediation via perceived autonomy and assertiveness $\left(a_{3} b_{3}=0.132, p=\right.$ 0.048). We could thus state that the non-negative effect of a candidate's transgender identity on their hiring chances is explained by the fact that the negative impact of co-workers' and customers' taste to collaborate is compensated by the positive impact of the perceived autonomy and assertiveness of a transgender job candidate.

As stated before, our main goal was to explore different potential explanations for hiring discrimination towards transgender men by investigating what exactly is being signalled by one's gender identity to employers in the hiring process. An additional goal from our study was, however, to identify different moderators of hiring discrimination towards transgender men. This will be the subject of the next subsection.

\subsection{Moderators of hiring discrimination against transgender men}

As previously shown in Subsection 3.1, we did not find any significant evidence for hiring discrimination against transgender men at the level of our full sample. However, this does not mean there is no hiring discrimination apparent among particular subsamples. As mentioned in Subsection 2.2, hiring discrimination may indeed be moderated by different participant characteristics and the occupation of the vacancy filled. To investigate the potential moderation 
effects of the different occupation and participant characteristics, we run two-tailed t-tests (supra Subsection 2.2). The results are presented in Table 4.

\section{< TABLE 4 ABOUT HERE >}

Panel A of Table 4 presents the difference in hiring probability by gender identity for three subsamples of our data by the occupation of the filled vacancy. The differences in hiring probability between the transgender and cisgender candidate in the three occupations are very similar and insignificant in all three cases. So, we do not find evidence for moderation effects in this respect.

Further, Panel B of Table 4 presents the potential moderation effects at the recruiters' side. First, we expected hiring discrimination towards transgender men to be higher among participants who are more risk-averse (Baert, 2018b). As shown in the aforementioned panel, we see this is not the case. On the contrary, we find a weakly significant difference (significant at the $10 \%$ level) in the evaluation of the transgender and cisgender candidate regarding their hiring probability in the two subsamples divided by risk aversion, where the participants who are less risk-averse rate cisgender men more positively than transgender men and more riskaverse participants score transgender men more positively than cisgender men. We thus find the opposite of what we expected. However, it should be acknowledged that this finding could be influenced by social desirability. More risk-averse participants are namely expected to answer in a more socially desirable way. Therefore, we control for the effect of social desirability and find that the weakly significant moderation effect of risk aversion disappears.

Second, hiring discrimination against transgender men can be expected to be higher among individuals with higher conformity to classical gender roles (Kerns and Fine, 1994; Worthen, 2013). However, in our study, we did not find any statistical differences in ratings of the hireability scale between the subsample with participants with low scores on the conformity to classical gender roles scale and the subsample with participants with high scores on this scale.

Third, contact with transgender individuals is expected to lead to more positive attitudes towards them, as well as to break negative stereotypes against them (Bukhari et al., 2016). As a consequence, lower levels of discrimination are expected with people who have had more contact with transgender individuals. Fourth, for this reason, and for a feeling of commonality, lesbian, gay, and bisexual individuals may also discriminate less against transgender individuals (Stone, 2009). However, looking at the results in Panel B of Table 4, this is not the case in our experiment. Again, we do not find any statistical differences in ratings on the hireability scale between the subsample with participants who have a lot of contact with transgender people (homosexual or bisexual participants) and the subsample with participants who have few contact with transgender individuals (heterosexual participants).

Last, Panel B of Table 4 also shows the difference in hiring probability when making subsamples based on socio-economic background characteristics. In doing so, we find that 
female students rate the transgender candidate more positively than the cisgender counterpart, while male students do not prefer one above the other. A potential explanation for this result can be found in the concept of homosociality. Homosociality refers to the fact that people prefer individuals similar to themselves. Following this line of thought, it might be the case that female students might prefer transgender men because they were assigned the female sex at birth and therefore are perceived as more similar to the female students themselves (Bird, 1996; Holgersson, 2013). A remark that has to be made, however, is that the results are only weakly significant (i.e. at the $10 \%$ level). Therefore, we cannot rule out the possibility that we found this result by coincidence. In addition, further analysis shows that we cannot speak of moderation by age and maternal and paternal education level.

As mentioned in Subsection 3.4, we additionally ran a multivariate regression analysis with interaction terms. The conclusions found using this approach are very similar to the results mentioned above. The results of this analysis are available upon request.

\section{Conclusion}

The aim of this study was to offer an explanation for hiring discrimination against transgender men. To this end, we conducted a scenario experiment in which participants made a fictitious hiring decision regarding one out of three job vacancies (i.e. a male-dominated job, a genderneutral job or a female-dominated job). More importantly, the participants had to rate a transgender or a cisgender male candidate regarding different statements related to the economic discrimination theories of taste-based (Becker, 1957) and statistical discrimination (Arrow, 1973). In addition, the participants were surveyed with respect to different personal characteristics.

Where the participants in our experiment indicated to have no issues with collaborating with transgender men themselves, they did indicate to suppose that other employees and customers would have negative attitudes towards collaborating with transgender men. We thus find empirical support for co-worker and customer discrimination but not for employer discrimination. Furthermore, we found that transgender men were perceived as being in worse health, being more autonomous and assertive, and having a lower probability to go on parental leave compared with cisgender men, revealing evidence for (positive and negative) statistical discrimination. Of these different explanations, the participants' belief that employees and customers would not want to collaborate with transgender people, as well as, the perception about transgender people's assertiveness and autonomy, acted as main explanations for hiring discrimination towards transgender men. Next to this, we found no evidence for moderation effects of (i) the gender type of a job, (ii) risk aversion, (iii) conformity to classical gender roles, (iv) contact with transgender people, (v) sexual orientation, and (vi) several personal background characteristics on hiring probability. Our results are, therefore, very similar to the ones of Van Borm and Baert (2018), mentioned in Section 1.

This study is innovative for being one of the first to explore the relative empirical importance of dominant explanations for hiring discrimination towards transgender individuals. Where 
previous research has mainly focused on measuring hiring discrimination towards transgender people, we focus on explaining it by investigating the mechanisms underlying the discrimination, therefore, taking the next step in the literature. By supplementing the existing studies of Reed et al. (2015) and Van Borm and Baert (2018), we are able to compare the results regarding transgender women with those of transgender men and get a more complete view on why hiring discrimination towards transgender individuals occurs (mediators) and in which situations this discrimination is higher (moderators). These insights are necessary to develop adequate policy actions and tackle the severe problem of hiring discrimination towards transgender individuals.

We end this article by acknowledging some research limitations and making a few suggestions for future research. First, as we mimicked the experimental design of Van Borm and Baert (2018), the limitations of their design also limit our conclusions. More concretely, the experimental setting of this type of research makes it difficult to know whether the dynamics measured in the lab will be (of) the same (magnitude) in the field. Because our participants knew they were participating in an experiment, it is possible they answered in a more socially desirable way compared with when they are unaware of their participation in an experiment (e.g. in a correspondence test). However, we did control for socially desirable answering in our study by excluding the participants with a high score on the social desirability scale from our research sample and found no significant differences in the results. Besides this, it has been shown that decisions made in scenario experiments highly correlate with actual behaviour (Baert and De Pauw, 2014; Van Belle et al., 2018).

In addition, given the fact that we use one specific profile which only varies with respect to the candidates' gender identity over the different resumes, our experimental design prevents us from investigating whether additional information on the resumes could influence the signals of one's transgender identity. It could, for example, be interesting to investigate whether the negative signal regarding a transgender candidate's health could be compensated by adding, for example, different sports activities on the candidate's resume.

Next to the experimental setting and design, the fact that we used students instead of real recruiters as participants could also bias our results because students might answer differently from real recruiters. However, by using final-year students enrolled in study programmes related to Human Resource Management, we limit this potential bias because these students should have the appropriate background knowledge to make rational and well-thought evaluations of job candidates. Moreover, previous studies show that students' evaluations do not largely diverge from those made by professional recruiters, especially when it comes to evaluating job candidates (Baert, 2018b; Falk et al., 2013; Hosoda et al., 2003). Nevertheless, future research could replicate the experiment using a sample of real recruiters as participants to check whether the evaluations of students are indeed similar to the one's of real recruiters.

A last limitation linked to mimicking the experimental design of Van Borm and Baert (2018) is the fact that our results are very hard to generalise to a broader context because our participants had to make recruitment decisions about openly transgender individuals who had a specific profile and had applied for one out of three specific occupations in Belgium. Future research should, therefore, replicate the study in different contexts. More precisely, it would be 
interesting to see whether the explanations for hiring discrimination towards transgender men (and women) differ in countries with less tolerant public opinions towards transgender persons. It could be that the compensating mechanism of the positive stereotype regarding transgender people assertiveness and autonomy does not play such a significant role in less tolerant countries. In addition, given that we find participants to suppose that other employees and customers would not like to collaborate with transgender individuals, it would be interesting to investigate whether hiring discrimination towards transgender individuals is higher in jobs associated with teamwork or more customer contact.

Second, as for all empirical studies, our statistical analyses are limited by the finite size of our research sample. As a consequence, some subsamples were too small to draw any straightforward conclusions regarding the heterogeneity in hiring discrimination by some of the participant characteristics, among which participants with a non-Belgian nationality and participants with a homosexual or bisexual sexual orientation. A larger research sample could have enabled us to provide more insights into the moderators of hiring discrimination towards transgender men. It would, therefore, be interesting to replicate the study with a larger research sample. 


\section{References}

Arrow, K.J. (1973), “The theory of discrimination”, in Ashenfelter, O. and Rees, A. (Eds.), Discrimination in Labor Markets, Princeton University Press, Princeton, pp. 3-33.

Badgett, M.V., Lau, H., Sears, B., and Ho, D. (2007), Bias in the workplace: Consistent evidence of sexual orientation and gender identity discrimination, The Williams Institute, Los Angeles, LA.

Baert, S. (2014), "Career lesbians. Getting hired for not having kids?", Industrial Relations Journal, Vol. 45 No. 6, pp. 543-561.

Baert, S. (2018a), "Hiring discrimination: an overview of (almost) all correspondence experiments since 2005", in Gaddis, S. (Ed.), Audit Studies: Behind the Scenes with Theory, Method, and Nuance, Methodos Series 14, Springer, Cham, pp. 63-77.

Baert, S., (2018b), "Hiring a gay man, taking a risk?: A lab experiment on employment discrimination and risk aversion", Journal of homosexuality, Vol 65 No. 8, pp. 1015-1031.

Baert, S., Aers, L., Bogaert, S., and Deschacht, N. (2019), “Does parenthood damage your cv?", Unpublished manuscript.

Baert, S. and De Pauw, A.S. (2014), "Is ethnic discrimination due to distaste or statistics?", Economics Letters, Vol. 125 No. 2, pp. 270-273.

Bardales, N. (2013), "Finding a job in 'a beard and a dress': Evaluating the effectiveness of transgender anti-discrimination laws”, Unpublished manuscript.

Becker, G.S. (1957), The Economics of Discrimination, University of Chicago Press, Chicago, IL.

Bird, S. R. (1996), "Welcome to the men's club: Homosociality and the maintenance of hegemonic masculinity", Gender and society, Vol. 10 No. 2, pp. 120-132.

Blais, A. and Weber, E. U. (2006), “A Domain-Specific Risk-Taking (DOSPERT) Scale for adult populations”, Judgment and Decision Making, Vol. 1 No. 1.

Blakemore, J.E.O. (2003), "Children's beliefs about violating gender norms: Boys shouldn't look like girls, and girls shouldn't act like boys", Sex Roles, Vol. 48 No. 9/10, pp. 411-419.

Bockting, W. (2014), "The impact of stigma on transgender identity development and mental health", in Kreukels, B. P. C., Steensma, T. D., and de Vries, A. L. C. (Eds.), Focus on sexuality research. Gender dysphoria and disorders of sex development: Progress in care and knowledge, Springer Science + Business Media, New York, NY, pp. 319-330.

Bockting, W. O., Miner, M. H., Swinburne Romine, R. E., Hamilton, A., and Coleman, E. (2013), "Stigma, mental health, and resilience in an online sample of the US transgender population”, American journal of public health, Vol. 103 No. 5, pp. 943-951. 
Beretvas, S.N., Meyers, J.L., and Leite, W.L. (2002), “A reliability generalization study of the Marlowe-Crowne social desirability scale", Educational and Psychological Measurements, Vol. 62 No. 4, pp. 570-589.

Bukhari, S., Mushtaq, H., and Aurangzaib, S. (2016), “Attitudes towards transgender: A study of gender and influencing factors", Journal of Gender and Social Issues, Vol. 15 No. 2, pp. 93-112.

Carpenter, C.S., Eppink, S.T., and Gonzales, G. (2016), "Transgender status, employment, and income", Unpublished manuscript.

Clements-Nolle, K., Marx, R., and Katz, M. (2006), "Attempted suicide among transgender persons: The influence of gender-based discrimination and victimization", Journal of homosexuality, Vol. 51 No. 3, pp. 53-69.

De Sutter, P. (2001), "Gender reassignment and assisted reproduction. Present and future reproductive options for transgender people", Human Reproduction, Vol. 16 No. 4, pp. $612-614$.

Drydakis, N. (2009), "Sexual orientation discrimination in the labour market", Labour Economics, Vol. 16 No. 4, pp. 364-372.

Drydakis, N. (2014), "Sexual orientation discrimination in the Cypriot labour market: Distastes or uncertainty?”, International Journal of Manpower, Vol. 35 No. 5, pp. 720-744.

Drydakis, N. (2015), "Measuring sexual orientation discrimination in the UK's labour market: A field experiment", Human Relations, Vol. 68 No. 11, pp. 1769-1796.

Drydakis, N. (2017a), “Trans employees, transitioning, and job satisfaction”, Journal of Vocational Behavior, Vol. 98 No. 1, pp. 1-16.

Drydakis N. (2017b), "Trans people, well-being, and labor market outcomes", IZA World of Labor, pp. 386-399.

Falk, A., Meier, S., and Zehnder, C. (2013), "Do lab experiments misrepresent social preferences? The case of self-selected student samples", Journal of the European Economic Association, Vol. 11 No. 4, pp. 839-852.

FRA. (2014), Being Trans in the European Union: Comparative Analysis of EU LGBT Survey Data, European Union Agency for Fundamental Rights, Vienna.

Geijtenbeek, L. and Plug, E. (2018), "Is there a penalty for registered women? Is there a premium for registered men? Evidence from a sample of transsexual workers", European Economic Review, Vol. 109, pp. 334-347.

Gorsuch, M. M. (2019), "Gender, sexual orientation, and behavioral norms in the labor market”, ILR Review, Vol. 72 No. 4, pp. 927-954.

Granberg, M., Ahmed, A., and Andersson, A.P. (2019), "Hiring discrimination against transgender people: Evidence from a field experiment”, Unpublished manuscript.

Grant, J.M., Mottet, L., Tanis, J.E., Harrison, J., Herman, J., and Keisling, M., (2011), Injustice at every turn: A report of the national transgender discrimination survey, National Center for Transgender Equality, Washington, DC. 
Hayes, A. F. (2013), Introduction to Mediation, Moderation, and Conditional Process Analysis: A Regression-Based Approach, The Guilford Press, New York, NY.

Heilman, M.E. (1983), "Sex bias in work settings: The lack of fit model”, in Staw, B. and Cummings, L. (Eds.), Research in Organizational Behavior (Vol 5), JAI Press, Greenwich, CT, pp. 269-298.

Hill, D. B. and Willoughby, B. L. (2005), "The development and validation of the genderism and transphobia scale", Sex roles, Vol. 53 No. 7, pp. 531-544.

Holgersson, C. (2013), "Recruiting managing directors: Doing homosociality", Gender, Work and Organization, Vol. 20 No. 4, pp. 454-466.

Hosoda, M., Stone-Romero, E. F., and Coats, G. (2003), "The effects of physical attractiveness on job-related outcomes: A meta-analysis of experimental studies", Personnel psychology, Vol. 56 No. 2, pp. 431-462.

Hughto, J.M.W., Reisner, S.L., and Pachankis, J.E. (2015), “Transgender stigma and health: A critical review of stigma determinants, mechanisms, and interventions", Social science and medicine, Vol. 147, pp. 222-231.

ILGA-Europe. (2019), Annual review of the human rights situation of lesbian, gay, bisexual, trans and intersex people 2019, ILGA-Europe, Brussels.

James, S., Herman, J., Rankin, S., Keisling, M., Mottet, L., and Anafi, M.A. (2016), The report of the 2015 US transgender survey, National Center for Transgender Equality, Washington, DC.

Kerns, J.G. and Fine, M.A. (1994), "The relation between gender and negative attitudes toward gay men and lesbians: Do gender role attitudes mediate this relation?", Sex Roles, Vol. 31 No. 5/6, pp. 297-307.

King, M. E., Winter, S., and Webster, B. (2009), "Contact reduces transprejudice: A study on attitudes towards transgenderism and transgender civil rights in Hong Kong”, International Journal of Sexual Health, Vol. 21 No.1, pp. 17-34.

Landén, M. and Innala, S. (2000), "Attitudes toward transsexualism in a Swedish national survey”, Archives of Sexual Behavior, Vol. 29 No. 4, pp. 375-388.

Levy, G. D., Taylor, M. G., and Gelman, S. A. (1995), "Traditional and evaluative aspects of flexibility in gender roles, social convention, moral rules, and physical laws", Child Development, Vol. 66 No. 2, pp. 515-531.Make the Road New York. (2010), Transgender need not apply: A report on gender identity job discrimination, Make the Road New York, New York, NY.

Motmans, J., De Biolley, I., and Debunne, S. (2009), Leven als transgender in België: de sociale en juridische situatie van transgender personen in kaart gebracht, Instituut voor de gelijkheid van vrouwen en mannen, Brussel.

Motmans, J., Wyverkens, E., and Defreyne, J., (2017), Leven als transgender persoon in België: 10 jaar later, Instituut voor de gelijkheid van vrouwen en mannen, Brussel. 
Nagoshi, J. L., Adams, K. A., Terrell, H. K., Hill, E. D., Brzuzy, S., and Nagoshi, C. T. (2008), "Gender differences in correlates of homophobia and transphobia", Sex Roles, Vol. 59, pp. 521-531.

Newsom, J. T., Prigerson, H. G., Schulz, R., and Reynolds III, C. F. (2003), "Investigating moderator hypotheses in aging research: Statistical, methodological, and conceptual difficulties with comparing separate regressions", The International Journal of Aging and Human Development, Vol. 57 No. 2, pp. 119-150.

Norton, A. T. and Herek, G. M. (2013), "Heterosexuals' attitudes toward transgender people: Findings from a national probability sample of U.S. adults", Sex Roles, Vol. 68 No. 11/12, pp. 738-753. Paris.

OECD. (2019), Society at a Glance 2019: OECD Social Indicators, OECD Publishing,

Padavic, I. and Reskin, B. (2002), Women and Men at Work, Pine Forge Press, Thousand Oaks, CA.Rainey, T., Imse, E., and Pomerantz, A. (2015), Qualified and transgender: A report on results of resume testing for employment discrimination based on gender identity, Office of Human Rights District of Columbia, Washington, DC.

Reed, O.M., Franks, A.S., and Scherr, K.C. (2015), “Are perceptions of transgender individuals affected by mental illness stigma? A moderated mediation analysis of antitransgender prejudice in hiring recommendations", Psychology of sexual orientation and gender diversity, Vol. 2 No. 4, pp. 463-469.

Reynolds, W. M. (1982), "Development of reliable and valid short forms of the Marlowe- Crowne Social Desirability Scale”, Journal of Clinical Psychology, Vol. 38 No. 1, pp. 119-125.

Sârbescu, P., Costea, I., and Rusu, S. (2011), "Psychometric properties of the MarloweCrowne social desirability scale in a Romanian sample", Procedia-Social and Behavioral Sciences, Vol. 33, pp. 707-711.

Schilt, K. and Wiswall, M. (2008), "Before and after: Gender transitions, human capital, and workplace experiences", The BE Journal of Economic Analysis and Policy, Vol. 8 No. 1, pp. 1935-1682.

Spence, J. T., Helmreich, R., and Stapp, J., (1973), "A short version of the Attitudes toward Women Scale (AWS)", Bulletin of the Psychonomic Society, Vol. 2 No. 4, pp. 219-220.

Stone, A. L., (2009), "More than adding a T: American lesbian and gay activists' attitudes towards transgender inclusion", Sexualities, Vol. 12 No. 3, pp. 334-354.

Stone-Romero, E. F., and Anderson, L. E. (1994), "Relative power of moderated multiple regression and the comparison of subgroup correlation coefficients for detecting moderating effects", Journal of applied psychology, Vol. 79 No. 3, pp. 354-359.

UNESCO. (2016), Out In the Open. Education Sector Responses to Violence Based on Sexual Orientation and Gender Identity/expression: Summary Report, UNESCO, Paris.

Valfort, M. (2017), LGBTI in OECD Countries, OECD Publishing, Paris. 
Van Belle, E., Di Stasio, V., Caers, R., De Couck, M., and Baert, S. (2018), "Why are employers put off by long spells of unemployment?", European Sociological Review, Vol. 34 No. 6, pp. 694-710.

Van Borm, H. and Baert, S., (2018), "What drives hiring discrimination against transgenders?", International Journal of Manpower, Vol.39 No. 4, pp. 581-599.

Van Hoye, G. and Lievens, F. (2003), "The effects of sexual orientation on hirability ratings: An experimental study”, Journal of Business and Psychology, Vol. 18 No. 1, pp. 1530 .

Weichselbaumer, D. (2015), "Testing for discrimination against lesbians of different marital status: A field experiment”, Industrial Relations, Vol. 54 No. 1, pp. 131-161.

West, K. and Hewstone, M. (2012), "Culture and contact in the promotion and reduction of anti-gay prejudice: Evidence from Jamaica and Britain”, Journal of Homosexuality, Vol. 59 No. 1, pp. 44-66.

Worthen, M.G. (2013), “An argument for separate analyses of attitudes toward lesbian, gay, bisexual men, bisexual women, MtF and FtM transgender individuals", Sex Roles, Vol. 68 No. 11/12, pp. 703-723.

Zucker, K. J., Wilson-Smith, D. N., Kurita, J. A., and Stern, A. (1995). “Children's appraisals of sex-typed behavior in their peers", Sex Roles, Vol. 33 No. 11/12, pp. 703-725. 
Appendix

<TABLE A1 ABOUT HERE >

<TABLE A2 ABOUT HERE > 


\section{Table 1. Data Description}

\begin{tabular}{|c|c|c|c|}
\hline & (1) & 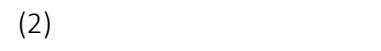 & (3) \\
\hline & Cis male candidate & Transgender male candidate & \\
\hline & $\begin{array}{l}\text { Mean } \\
n=126\end{array}$ & $\begin{array}{l}\text { Mean } \\
n=126\end{array}$ & Difference: $(2)-(1)$ \\
\hline \multicolumn{4}{|l|}{ A. Manipulation check } \\
\hline 'The candidate was born as a woman' & 1.500 & 6.532 & $5.032 * * *[-31.153]$ \\
\hline \multicolumn{4}{|l|}{ B. Occupation } \\
\hline Management assistant & 0.349 & 0.349 & $0.000[0.000]$ \\
\hline Logistics clerk & 0.325 & 0.333 & $0.008[-0.133]$ \\
\hline Site manager & 0.325 & 0.317 & $-0.008[0.134]$ \\
\hline \multicolumn{4}{|l|}{ C. Participant characteristics } \\
\hline Male gender & 0.421 & 0.476 & $0.056[-0.884]$ \\
\hline Age & 21.726 & 22.040 & $0.314 *[-1.925]$ \\
\hline Foreign nationality & 0.000 & 0.032 & $0.032 * *[-2.025]$ \\
\hline Father with tertiary education & 0.508 & 0.557 & $0.049[-0.773]$ \\
\hline Mother with tertiary education & 0.653 & 0.677 & $0.024[-0.402]$ \\
\hline Lesbian, gay, or bisexual sexual orientation & 0.056 & 0.064 & $0.007[-0.249]$ \\
\hline Risk aversion scale & 3.606 & 3.720 & $0.114[-1.032]$ \\
\hline Conformity to classical gender roles scale & 3.710 & 3.668 & $-0.042[1.005]$ \\
\hline Contact with trans persons scale & 1.313 & 1.336 & $0.022[-0.283]$ \\
\hline Social desirability bias scale & 0.580 & 0.548 & $-0.033[1.449]$ \\
\hline
\end{tabular}


Table 2. Bivariate Analysis of Candidate Evaluation Scores

\begin{tabular}{|c|c|c|c|}
\hline & (1) & (2) & (3) \\
\hline & Cis male candidate & Transgender male candidate & \\
\hline & $\begin{array}{l}\text { Mean } \\
n=126\end{array}$ & $\begin{array}{l}\text { Mean } \\
n=126\end{array}$ & Difference: (2) - (1) \\
\hline \multicolumn{4}{|l|}{ A. Interview and hiring probability } \\
\hline 'I will invite the candidate for a job interview' & 5.889 & 5.857 & $-0.032[0.256]$ \\
\hline 'I will hire the job candidate’ & 4.976 & 5.059 & $0.83[-0.639]$ \\
\hline \multicolumn{4}{|c|}{ B. Candidate evaluation scales related to the models of taste-based and statistical discrimination } \\
\hline ‘As an employer, I will enjoy collaborating with this candidate' & 5.048 & 5.004 & $-0.044[0.369]$ \\
\hline 'My co-workers will enjoy collaborating with this candidate' & 5.000 & 4.412 & $-0.588 * * *[4.602]$ \\
\hline 'My customers will enjoy collaborating with this candidate' & 4.889 & 4.440 & $-0.448 * * *[3.350]$ \\
\hline 'This candidate will provide sufficient autonomy' & 4.944 & 5.413 & $0.468 * * *[-3.595]$ \\
\hline 'This candidate will provide sufficient assertiveness' & 4.841 & 5.238 & $0.397 * * *[-3.021]$ \\
\hline 'This candidate will be paternity leave in the short or medium run' & 2.944 & 2.444 & $-0.500 * * *[3.041]$ \\
\hline 'This candidate will be on sick leave in the short or medium run' & 2.700 & 3.262 & $0.563 * * *[-3.338]$ \\
\hline
\end{tabular}

Notes. See Subsection 2.1.2 for a description of the candidate evaluation scales. See Subsection 3.2 for a discussion of the results. All candidate evaluation statements are scored on a 7-point Likert scale. $T$-tests are performed to test whether the differences presented in Column (3) are significantly different from $0 .{ }^{* * *}(* *)((*))$ indicates significance at the $1 \%(5 \%)((10 \%))$ significance level. $T$-statistics are between brackets. 
Table 3. Correlations Between Candidate Evaluation Scores

\begin{tabular}{|c|c|c|c|c|c|c|c|c|c|}
\hline & (1) & $(2)$ & (3) & (4) & (5) & (6) & (7) & $(8)$ & (9) \\
\hline (1) 'I will invite the candidate for a job interview' & 1.000 & & & & & & & & \\
\hline (2) 'I will hire the job candidate' & $0.609 * * *$ & 1.000 & & & & & & & \\
\hline (3) 'As an employer, I will enjoy collaborating with this candidate' & $0.387 * * *$ & $0.478^{* * *}$ & 1.000 & & & & & & \\
\hline (4) 'My co-workers will enjoy collaborating with this candidate' & $0.263^{* * *}$ & $0.373^{* * *}$ & $0.544^{* * *}$ & 1.000 & & & & & \\
\hline (5) 'My customers will enjoy collaborating with this candidate' & $0.306^{* * *}$ & $0.444^{* * *}$ & $0.587^{* * *}$ & $0.691 * * *$ & 1.000 & & & & \\
\hline (6) 'This candidate will provide sufficient autonomy' & $0.386^{* * *}$ & $0.494 * * *$ & $0.383^{* * *}$ & $0.202^{* * *}$ & $0.377^{* * *}$ & 1.000 & & & \\
\hline (7) 'This candidate will provide sufficient assertiveness' & $0.350 * * *$ & $0.338^{* * *}$ & $0.385^{* * *}$ & $0.192^{* * *}$ & $0.268^{* * *}$ & $0.577^{* * *}$ & 1.000 & & \\
\hline (8) 'This candidate will be on paternity leave in the short or medium run' & -0.039 & -0.018 & 0.024 & 0.104 & $0.110^{*}$ & -0.089 & 0.009 & 1.000 & \\
\hline (9) 'This candidate will be on sick leave in the short or medium run' & -0.040 & $-0.108^{*}$ & -0.035 & -0.057 & $-0.116^{*}$ & -0.055 & -0.021 & $0.280 * * *$ & 1.000 \\
\hline
\end{tabular}

Notes. Correlation coefficients are reported. See Subsection 3.2 for a discussion of the results. ${ }^{* * *}\left({ }^{* *}\right)\left(\left({ }^{*}\right)\right)$ indicates significance at the $1 \%(5 \%)((10 \%))$ significance level. These statistics are based on the full sample of 252 evaluations. 
Figure 1. Multiple Mediation Model

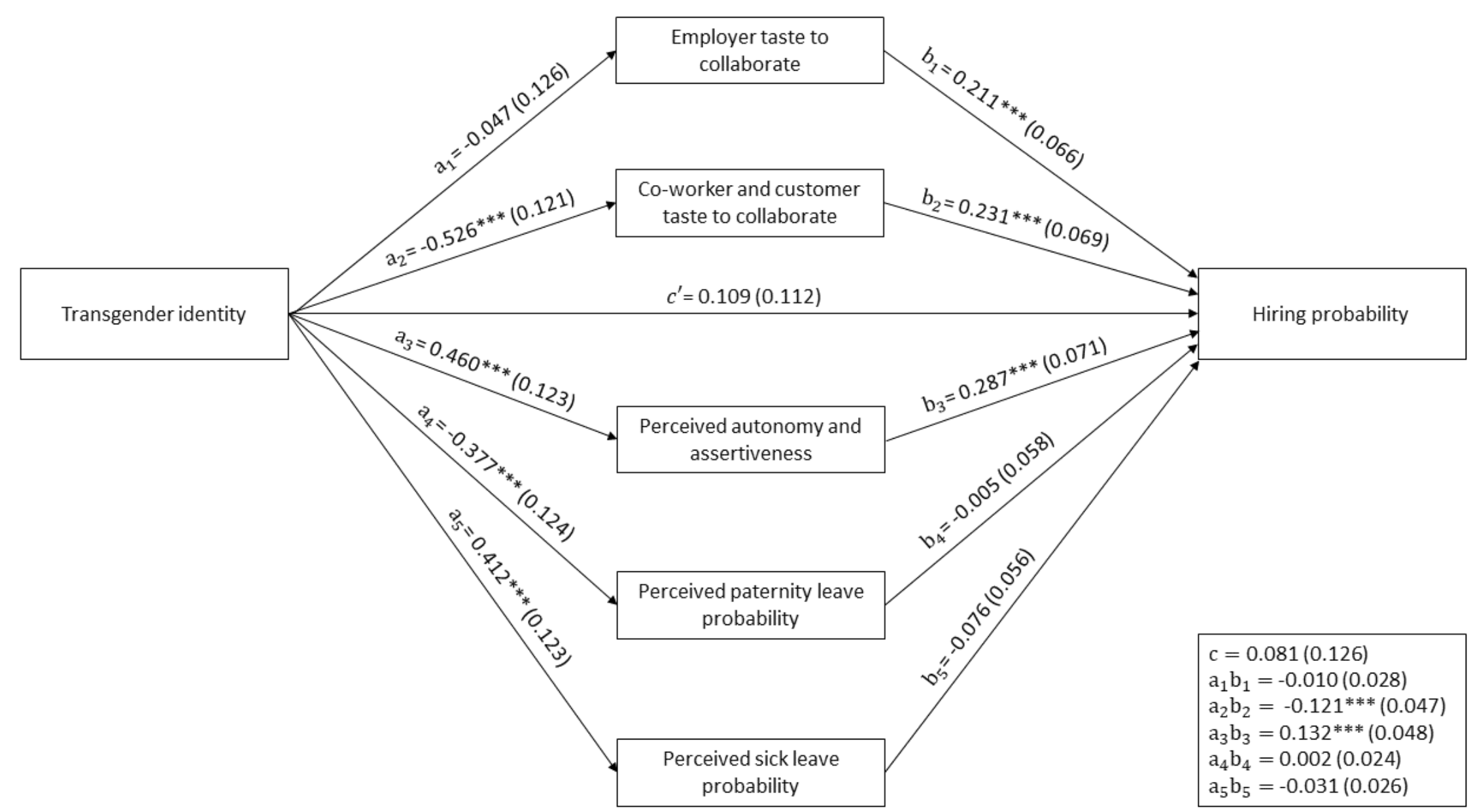

Notes. The presented statistics are coefficient estimates (and standard errors in parentheses) for the mediation model outlined in Subsection 3.3. $\mathrm{c}$ stands for the total effect, $\mathrm{c}^{\prime}$ for the direct effect, and aibi for the indirect effects of a transgender status on the likelihood of a positive hiring decision passing through a mediator $M_{i}$. Standard errors are corrected for clustering of the observations at the participant level. The confidence intervals for the mediation effects are based on 1,000 bootstrap samples. ${ }^{* * *}\left({ }^{* *}\right)((*))$ indicates significance at the $1 \%(5 \%)((10 \%))$ significance level. 
Table 4. Hiring Probability by Occupation and Participant Characteristics

\begin{tabular}{|c|c|c|c|c|c|}
\hline & (1) & (2) & (3) & (4) & (5) \\
\hline & \multicolumn{2}{|c|}{ Cis male candidate } & \multicolumn{3}{|c|}{ Transgender male candidate } \\
\hline & $n$ & $\begin{array}{l}\text { Mean hiring } \\
\text { probability }\end{array}$ & $n$ & $\begin{array}{l}\text { Mean hiring } \\
\text { probability }\end{array}$ & Difference: (4) - (2) \\
\hline \multicolumn{6}{|l|}{ A. Subsamples by occupation } \\
\hline Management assistant & 44 & 4.773 & 44 & 4.909 & $0.136[-0.548]$ \\
\hline Logistics clerk & 41 & 5.024 & 42 & 5.179 & $0.154[-0.762]$ \\
\hline Site manager & 41 & 5.146 & 40 & 5.100 & $-0.046[0.212]$ \\
\hline \multicolumn{6}{|l|}{ B. Subsamples by candidate characteristics } \\
\hline Female gender & 73 & 5.096 & 66 & 5.356 & $0.260 *[-1.685]$ \\
\hline Male gender & 53 & 4.811 & 60 & 4.733 & $-0.078[0.369]$ \\
\hline Age equal to or below 22 (median) & 102 & 4.912 & 95 & 5.023 & $0.230[-1.574]$ \\
\hline Age above 22 (median) & 24 & 5.250 & 31 & 4.806 & $-0.443[1.568]$ \\
\hline Father with less than tertiary education & 61 & 5.016 & 54 & 5.083 & $0.067[-0.316]$ \\
\hline Father with tertiary education & 63 & 4.936 & 68 & 5.059 & $0.122[-0.727]$ \\
\hline Mother with less than tertiary education & 43 & 5.139 & 40 & 5.412 & $0.273[-1.115]$ \\
\hline Mother with tertiary education & 81 & 4.889 & 84 & 4.893 & $0.004[-0.026]$ \\
\hline Heterosexual orientation & 117 & 4.966 & 117 & 5.077 & $0.111[-0.815]$ \\
\hline Lesbian, gay, or bisexual orientation & 7 & 5.143 & 8 & 4.812 & $-0.330[0.643]$ \\
\hline Risk aversion scale equal or below 3.667 (median) & 73 & 5.014 & 62 & 4.839 & $-0.175[0.956]$ \\
\hline Risk aversion scale above 3.667 (median) & 53 & 4.924 & 64 & 5.273 & $0.349 *[-1.902]$ \\
\hline Conformity to classical gender roles scale equal to or below 3.667 (median) & 61 & 5.000 & 68 & 5.043 & $0.043[-0.234]$ \\
\hline Conformity to classical gender roles scale above 3.667 (median) & 65 & 4.954 & 56 & 5.080 & $0.126[-0.672]$ \\
\hline Contact with trans persons scale equal to or below 1.000 (median) & 82 & 4.939 & 78 & 5.051 & $0.112[-0.621]$ \\
\hline Contact with trans persons scale above 1.000 (median) & 44 & 5.045 & 48 & 5.073 & $0.027[-0.160]$ \\
\hline
\end{tabular}

Notes. See Subsection 2.1.3 for a description of the participant characteristic scales. See Subsection 3.2 for a discussion of the results. For some of the breakdowns in Panel B, the total number of observations does not equal 252 due to missing values for some of the candidate characteristics. $T$-tests are performed to test whether the differences presented in Column (3) are significantly different from $0 .{ }^{* * *}\left({ }^{* *}\right)\left(\left(^{*}\right)\right)$ indicates significance at the $1 \%(5 \%)((10 \%))$ significance level. T-statistics are between brackets. The results regarding the participants' nationality are excluded from the table because the distribution of the fictitious profiles over Belgian and foreign participants was not balanced. 
Table A1. Vacancy Description

\begin{tabular}{|c|c|c|c|}
\hline & Management assistant & Logistics clerk & Site manager \\
\hline Job description & $\begin{array}{l}\text { This employee provides support to the management } \\
\text { team and is responsible for: } \\
\text { - Managing the agendas of the management and co- } \\
\text { ordinating the management activities. } \\
\text { - General administration: preparing presentations } \\
\text { and official documents, taking note of meetings, } \\
\text { drafting the internal planning, and contacting } \\
\text { suppliers. } \\
\text { - Preparing correspondence documents. } \\
\text { - Daily follow-up and filtering of letters, emails, faxes, } \\
\text { and phone calls. }\end{array}$ & $\begin{array}{l}\text { This employee is responsible for the processing and } \\
\text { follow-up of all kinds of logistics assignments: } \\
\text { - Coordinating and executing transport assignments. } \\
\text { - Receiving and registering incoming orders. } \\
\text { - Registration of transport operations. } \\
\text { - Creating logistics files. } \\
\text { - Tracking shipments on their way to their final } \\
\text { destination. } \\
\text { - Informing customers about their shipments. } \\
\text { - Administrative follow-up of import and export files. }\end{array}$ & $\begin{array}{l}\text { This employee is responsible for the daily management } \\
\text { at the building yard and is responsible for: } \\
\text { - Preparing, coordinating and following up the work } \\
\text { on the yard. } \\
\text { - Equipment management, administration of } \\
\text { deliveries, and safety on the yard. } \\
\text { - Design of a correct staffing plan. } \\
\text { - Contacts with the customer and the preparation of } \\
\text { claim states. } \\
\text { - General site administration. }\end{array}$ \\
\hline Profile description & $\begin{array}{l}\text { The required qualifications for this job are: } \\
\text { - A tertiary education degree (Bachelor and/or } \\
\text { Master). } \\
\text { - At least three years of experience in a similar job. } \\
\text { - Good knowledge of Dutch, French, and English. } \\
\text { - Good knowledge of the Microsoft Office software } \\
\text { programmes Outlook, Word, and Excel. } \\
\text { - The employee works punctually and accurately. } \\
\text { - The employee is strong in organisational and } \\
\text { administrative tasks. } \\
\text { - The employee is a team player. }\end{array}$ & $\begin{array}{l}\text { The required qualifications for this job are: } \\
\text { - A tertiary education degree (Bachelor and/or } \\
\text { Master). } \\
\text { - At least three years of experience in a similar job. } \\
\text { - Good knowledge of Dutch, French, and English. } \\
\text { - Good knowledge of the Microsoft Office software } \\
\text { programmes Outlook, Word, and Excel. } \\
\text { - The employee works punctually and accurately. } \\
\text { - The employee is strong in organisational and } \\
\text { administrative tasks. } \\
\text { - The employee is a team player. }\end{array}$ & $\begin{array}{l}\text { The required qualifications for this job are: } \\
\text { - A tertiary education degree (Bachelor and/or } \\
\text { Master). } \\
\text { - At least three years of experience in a similar job. } \\
\text { - Good knowledge of Dutch, French, and English. } \\
\text { - Good knowledge of the Microsoft Office software } \\
\text { programmes Outlook, Word, and Excel. } \\
\text { - The employee works punctually and accurately. } \\
\text { - The employee is strong in organisational and } \\
\text { administrative tasks. } \\
\text { - The employee is a team player. } \\
\text { - The employee has managerial skills. }\end{array}$ \\
\hline
\end{tabular}

Notes. The descriptions presented in this table are literally adopted from Van Borm and Baert (2018). 
Table A2. Candidate Evaluation Scores Among Participants With Low Social Desirability Bias

\begin{tabular}{|c|c|c|c|}
\hline & 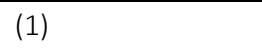 & $(2)$ & (3) \\
\hline & Cis male candidate & Transgender male candidate & \\
\hline & Mean & Mean & Difference: $(2)-(1)$ \\
\hline A. Participants with social desirability bias scale below 0.743 (mean + 1SD) & $n=96$ & $n=105$ & \\
\hline 'I will invite the candidate for a job interview' & 5.854 & 5.771 & $-0.083[0.578]$ \\
\hline 'I will hire the job candidate’ & 4.927 & 4.995 & $0.068[-0.457]$ \\
\hline 'As an employer, I will enjoy collaborating with this candidate' & 5.042 & 4.967 & $-0.075[0.571]$ \\
\hline 'My co-workers will enjoy collaborating with this candidate' & 4.937 & 4.361 & $-0.577 * * *[3.955]$ \\
\hline 'My customers will enjoy collaborating with this candidate' & 4.854 & 4.405 & $-0.449 * * *[3.027]$ \\
\hline 'This candidate will provide sufficient autonomy' & 4.885 & 5.381 & $0.495^{* * *}[-3.349]$ \\
\hline 'This candidate will provide sufficient assertiveness' & 4.792 & 5.171 & $0.380 * *[-2.577]$ \\
\hline 'This candidate will be on paternity leave in the short or medium run' & 2.844 & 2.400 & $-0.444 * *[2.419]$ \\
\hline 'This candidate will be on sick leave in the short or medium run' & 2.625 & 3.314 & $0.689 * * *[-3.685]$ \\
\hline B. Participants with social desirability bias scale below 0.564 (mean) & $n=60$ & $n=75$ & \\
\hline 'I will invite the candidate for a job interview' & 5.917 & 5.760 & $-0.157[0.921]$ \\
\hline 'I will hire the job candidate' & 4.900 & 4.953 & $0.053[-0.322]$ \\
\hline 'As an employer, I will enjoy collaborating with this candidate' & 5.050 & 4.967 & $-0.083[0.585]$ \\
\hline 'My co-workers will enjoy collaborating with this candidate' & 4.933 & 4.372 & $-0.562 * * *[3.261]$ \\
\hline 'My customers will enjoy collaborating with this candidate' & 4.867 & 4.380 & $-0.487 * * *[2.827]$ \\
\hline 'This candidate will provide sufficient autonomy' & 4.767 & 5.333 & $0.567^{* * *}[-3.317]$ \\
\hline 'This candidate will provide sufficient assertiveness' & 4.767 & 5.120 & $0.353^{* *}[-2.043]$ \\
\hline 'This candidate will be on paternity leave in the short or medium run' & 2.850 & 2.453 & $-0.397 *[1.683]$ \\
\hline 'This candidate will be on sick leave in the short or medium run' & 2.667 & 3.320 & $0.653 * * *[-2.858]$ \\
\hline
\end{tabular}

Notes. All candidate evaluation statements were scored on a 7-point Likert scale. T-tests are performed to test whether the differences presented in Column (3) are significantly different from $0 .{ }^{* * *}\left({ }^{* *}\right)\left(\left(^{*}\right)\right)$ indicates significance at the $1 \%(5 \%)((10 \%))$ significance level. $T$-statistics are between brackets. 